\title{
PROTECCIÓN DE DERECHOS EN SALUD EN EL PERÚ: EXPERIENCIAS DESDE EL ROL FISCALIZADOR DE LA SUPERINTENDENCIA NACIONAL DE SALUD
}

\author{
Óscar Quijano-Caballero1,3,a, Óscar Munares-García²,4,b
}

\begin{abstract}
RESUMEN
En Latinoamérica, la fiscalización y sanción en materia de salud es responsabilidad de las superintendencias nacionales. Por ello, sus sistemas normativos tienen la finalidad de proteger los derechos en salud, detectar sus vulneraciones y resolverlas. Este artículo discute el enfoque que el Perú ha asumido en derecho a la salud, entendida como un derecho programático y no fundamental, y respecto a la determinación de sanciones que determina. La función reguladora y fiscalizadora, esencial en salud pública, en nuestro país desde el 2015 está a cargo de la Superintendencia Nacional de Salud (SUSALUD) a través de la Intendencia de Fiscalización y Sanción. Se presenta la experiencia de SUSALUD, en la implementación del proceso administrativo sancionador (PAS), desde enero de 2014 a abril de 2016. En este período se determinó 38 PAS con responsabilidad. De éstas, 78,3\% corresponde a instituciones prestadoras de servicios de salud. Se concluye que las sanciones aplicadas por SUSALUD se derivan del derecho a la salud a nivel programático.
\end{abstract}

Palabras clave: Regulación y fiscalización en salud; Fiscalización sanitaria; Derechos del paciente (fuente: DeCS BIREME).

\section{PROTECTION OF HEALTH RIGHTS IN PERU: SUPERVISORY EXPERIENCES FROM THE VIEWPOINT OF THE NATIONAL HEALTH AUTHORITY}

\begin{abstract}
In Latin America, national superintendents are charged with the supervision and enforcement of health-related matters, and accordingly the regulatory systems used by these individuals are aimed at protecting health rights and detecting solving infringements. Here, we discuss the approach undertaken by Peru with regard to health rights as programmatic rather than fundamental rights, as well as the determination of penalties. Since 2015, the National Health Authority (SUSALUD), through the Control and Penalty Intendance, has maintained both the regulatory and supervisory roles deemed essential to public health in our country. Accordingly, we present the experiences of SUSALUD with regard to the implementation of the punitive administrative process (PAP) from January 2014 to April 2016. During this period, 38 PAP responsibilities were determined and of these, $78.3 \%$ correspond to institutions that provide health services. In conclusion, the penalties applied by SUSALUD are derived from programmatic-level health rights.
\end{abstract}

Key words: Health care coordination and monitoring; Sanitary supervision; Patient rights (source: MeSH NLM).

\section{FISCALIZACIÓN EN SALUD}

En Latinoamérica, las funciones de regulación y fiscalización en materia de salud son encargadas a las superintendencias, quienes se encargan de establecer reglas que rigen la interacción entre financiadores, prestadores y usuarios ${ }^{(1)}$. Según Robles ${ }^{(2)}$, dentro de las seis dimensiones que comprende el ejercicio de la rectoría en salud, la dimensión de regulación y fiscalización consiste en la capacidad y responsabilidad que tiene la Autoridad de
Salud Nacional para proponer, definir y establecer el marco legal y normativo, así como las políticas públicas en salud; y ello implica establecer las reglas y estándares oficiales de cumplimiento obligatorio.

Ensalud pública, el rol de fiscalización (supervisión deacciones en salud) y sanción (consecuencia del incumplimiento de la norma) forma parte de las funciones esenciales de la salud pública (FESP, específicamente el numeral 6). Las FESP son categorías operacionales que garantizan los procesos de

\footnotetext{
Intendencia de Fiscalización y Sanción, Superintendencia Nacional de Salud. Lima, Perú.

Intendencia de Investigación y Desarrollo, Superintendencia Nacional de Salud. Lima, Perú.

Facultad de Derecho y Ciencias Políticas, Universidad Nacional Mayor de San Marcos. Lima, Perú.

Facultad de Medicina Humana, Universidad Nacional Mayor de San Marcos. Lima, Perú.

Abogado; ${ }^{\text {b }}$ obstetra, doctor en Ciencias de la Salud

Recibido: 14/05/2016 Aprobado: 15/06/2016
}

Citar como: Quijano-Caballero O, Munares-García O. Protección de derechos en salud en el Perú: experiencias desde el rol fiscalizador de la Superintendencia Nacional de Salud. Rev Peru Med Exp Salud Publica. 2016;33(3)529-34. doi:10.17843/rpmesp.2016.333.2303 
trasformación e inserción del sistema de salud en un marco de protección de derechos ${ }^{(3)}$. El derecho a la salud ${ }^{(4)}$ representa un derecho humano fundamental que los estados deben garantizar y regular a través de sus entes rectores ${ }^{(5)}$. Copetta y Barrera ${ }^{(6)}$, en su informe sobre el financiamiento, regulación y fiscalización de la salud en Latinoamérica, refieren que operativamente, este rol se ha implementado a través de superintendencias: en Colombia por la Superintendencia Nacional de Salud (eminentemente judicializada); en Argentina por la Superintendencia de Servicios de Salud (también judicializada); en Brasil por la Agencia Nacional de Salud (que realiza notificaciones preliminares y opera mediante núcleos gestionados). En República Dominicana, esta función es desarrollada por la Superintendencia de Salud y Riesgos Laborales con responsabilidad de fiscalización, supervisión, conciliación y fiscalización en primera instancia, en Paraguay por la Superintendencia de Salud con rol fiscalizador y primer nivel de resolución de conflictos entre prestadoras de servicios de salud y aseguradoras. En México, la Dirección General de Calidad e Informática en la Comisión Nacional de Arbitraje Médico ofrece medios alternos para la solución de controversias entre usuarios y prestadores de servicios médicos, actuando como árbitro experto, imparcial, respetuoso y confidencial. Por último, en Ecuador el proceso de regulación está aun en estructuración.

Por otro lado, en Brasil, los servicios privados son regulados por laAgencia Nacional de Salud Suplementaria (ANS) y el Sistema Único de Salud es ejercido por el Departamento de Sistemas de Regulación Evaluación y Control. Ambas instancias realizan la investigación preliminar y tienen autoridad para desarrollar procesos administrativo para determinar infracciones, y aplicar sanciones en el ámbito de la ANS ${ }^{(6,7)}$. En Chile la Superintendencia de Salud vela por el cumplimiento de la normativa, fiscalizando a prestadores públicos y privados del sistema de salud ${ }^{(8)}$.

En Argentina, la Superintendencia de Servicios de Salud, redujo los indicadores de incidencia judicial en casi un $10 \%$ en un período de dos años. En Colombia durante el año 2007 , el $70 \%$ de las tutelas (reclamos de derechos fundamentales) ocurrieron en temas de salud. En ese país, la superintendencia impone multas y sanciones para el cumplimiento efectivo de las normas ${ }^{(6)}$.

En el Perú, la Superintendencia Nacional de Salud, (SUSALUD), ejerce la fiscalización y la función sancionadora de acciones que pueden afectar a usuarios de instituciones aseguradoras de fondos de aseguramiento en salud (IAFAS), de instituciones prestadoras de servicios de salud (IPRESS) y de unidades de gestión de instituciones prestadoras de servicios de salud (UGIPRESS). La sanción se ajusta al nivel de gravedad que las infracciones pueden ocasionar en los usuarios de los servicios de salud (leve, grave y muy grave). El proceso administrativo sancionador (PAS), identifica la existencia de una infracción, y si el caso lo requiere, determina una sanción ${ }^{(9,10)}$.

\section{LA SALUD COMO DERECHO}

Los derechos humanos han recibido diversas denominaciones a través del tiempo: derechos del hombre, garantías individuales o sociales, derechos naturales, derechos innatos, derechos esenciales, libertades públicas, derechos de la persona humana, derechos públicos subjetivos y derechos fundamentales ${ }^{(4)}$. Los derechos se singularizan de acuerdo a sus principios: universalidad, historicidad, progresividad, aspecto protector, indivisibilidad y eficacia directa. La Declaración Universal de los Derechos Humanos ${ }^{(11)}$ reconoce que: I... Toda persona tiene derecho a un nivel de vida adecuado que le asegure, así como a su familia, la salud y bienestar, y en especial la alimentación, el vestido, la vivienda, la asistencia médica y los servicios sociales necesarios... El Pacto Internacional de Derechos Económicos, Sociales y Culturales ${ }^{(12)}$, señala el derecho de toda persona al disfrute del más alto nivel posible de salud física y mental. Alcántara ${ }^{(13)}$ sostiene que la salud es un hecho social tanto material como inmaterial, por ello, es multidimensional y multicausal, desbordando así la competencia médica. Para Ferrajoli (14) los derechos fundamentales conforman tres criterios axiológicos: el nexo entre derechos humanos y paz (garantizando los derechos fundamentales para conservar la paz), el nexo entre derechos e igualdad (igual valoración para todos), y el papel de la ley del más débil en alternativa a la ley del más fuerte.

\section{DERECHO UNIVERSAL A LA SALUD: PROGRAMÁTICO Y FUNDAMENTAL}

El derecho a la salud surge como derecho universal de segunda generación, un derecho social de carácter programático; los cuales son derechos económicos y sociales guardando coherencia con lo establecido en la Constitución. Se definen como Obligaciones mediatas del Estado ${ }^{(12)}$, que necesitan de un proceso de ejecución de políticas sociales para que el ciudadano pueda gozar de ellos o ejercitarlos de manera plena. Los derechos programáticos no facultan a los ciudadanos a requerir judicialmente su ejecución inmediata, pues no gozarían de tutela jurisdiccional; sin embargo, estos derechos sí serían exigibles políticamente, y podrían encontrar satisfacción de acuerdo a la coyuntura gubernamental vigente. Ello se confirma con el tenor del Código Procesal Constitucional Peruano (15), que delimita el campo de aplicación de los procesos de amparo solo a afectaciones directas de los derechos fundamentales expresos o implícitos, o por trasgresiones a su ámbito constitucionalmente protegido. 
La concepción programática del derecho a la salud es recogida hoy por la mayoría de constituciones en el mundo; en Brasil, la Constitución de la República Federativa considera al derecho a la salud como derecho social; en Colombia, la Constitución considera a la salud como un derecho social y la atención de la salud un servicio público; en México, la Constitución de Querétaro indica que: Toda persona tiene derecho a la protección de la salud; en España, la Constitución reconoce el derecho a la protección de la salud; en Italia, la Constitución de la República señala la protección a la salud como derecho fundamental e interés básico de la colectividad. La Constitución Política del Perú contiene normas similares de orden programático. Su artículo 7. ${ }^{\circ}$ plantea que: Todos tienen derecho a la protección de su salud, la del medio familiar y la de la comunidad así como el deber de contribuir a su promoción y defensa y el Estado determina la política nacional de salud. El artículo 9. ${ }^{\circ}$ precisa que; El Poder Ejecutivo norma y supervisa su aplicación. Es responsable de diseñarla y conducirla en forma plural y descentralizadora para facilitar a todos el acceso equitativo a los servicios de salud.

Si el derecho a la salud es un derecho programático y no fundamental de las personas, el Estado no confiere tutela jurisdiccional. Algunas soluciones a la cuestión se han dado desde la jurisprudencia constitucional, en dos posiciones sustancialmente diferentes. Denominaremos enfoque conservador a la posición que subordina la protección constitucional del derecho a la salud a que este se encuentre vinculado con otros derechos fundamentales. En esta perspectiva el Tribunal Constitucional de España ${ }^{(16)}$, indica: /...El derecho a la salud o, mejor aun, el derecho a que no se dañe o perjudique la salud personal, queda comprendido en el derecho a la integridad personal. En similar posición, el Tribunal Constitucional del Perú, en la Sentencia 2016-2004AA/TC (17), precisa: /...Nuestro ordenamiento jurídico el derecho a la salud no se encuentra contemplado entre los derechos fundamentales formalmente establecidos en el Artículo $2{ }^{\circ}$ de la Constitución y, más bien, se le reconoce en el Capítulo de los Derechos Económicos y Sociales a que se refieren los Artículos 7. y 9. sin embargo, considera que cuando la vulneración del derecho a la salud compromete otros derechos fundamentales, como el derecho a la vida, la integridad física o el libre desarrollo de la personalidad, tal derecho acentúa su carácter fundamental $y$, por tanto, su afectación merece protección vía acción de amparo.

Los derechos fundamentales representan los pilares de un sistema jurídico, no existen derechos por encima de ellos, coexisten con otros derechos fundamentales en condiciones de igualdad; solamente para un caso concreto un derecho fundamental puede prevalecer sobre otro, previa aplicación del test de ponderación o proporcionalidad. En contraste el enfoque conservador propugna la validez de la protección constitucional al derecho fundamental a la salud por su vinculación con otro derecho fundamental -por ejemplo el derecho a la vida-. Es decir, subordina el derecho a la salud al derecho a la vida o a cualquiera con el que se le quisiera relacionar para activar su defensa. De acuerdo a este enfoque el derecho a la salud no consitituye un derecho fundamental en forma independiente sino en la medida en que se vincule con otro derecho.

La segunda respuesta al problema planteado viene desde la Corte Constitucional de Colombia, en una postura que llamaremos enfoque progresista. Éste considera al derecho a la salud como derecho fundamental de las personas, sin dejar de lado su esfera de derecho universal programático. En la Sentencia T-737/13 se dictamina: El derecho a la salud es un derecho fundamental de carácter autónomo. Según el artículo 49 de la Constitución Política, la salud tiene una doble connotación -derecho constitucional y servicio público- (...) siendo latente la amenaza de transgresión, el juez de tutela debe hacer efectiva su protección mediante este mecanismo, sin excepción. El derecho a la salud es un derecho fundamental y tutelable, que debe ser garantizado a todos los seres humanos igualmente dignos, siendo la acción de tutela el medio judicial más idóneo para defenderlo, en aquellos casos en los que la persona que requiere el servicio de salud es un sujeto de especial protección constitucional. En similar sentido, en la Sentencia T-760/08 indica: /...Se trata de derechos subjetivos de aplicación inmediata y la esencialidad e inalienabilidad del derecho para la persona.

En el Perú, independientemente del enfoque, ese desarrollo del derecho a la salud se aprecia en alguna jurisprudencia del Tribunal Constitucional, entre otras: Sentencia 2002-2006-PC/TC, caso Pablo Fabián Martínez y otros, que ordena al Ministerio de Salud implementar un sistema de emergencia para atender la salud de las personas contaminadas por plomo en La Oroya. Sentencias 2945-2003-AA/TC y 2016-2004AA/TC, casos Azanca Meza García y José Correa Condori, que ordenan al Ministerio de Salud brindar a los demandantes tratamiento contra el VIH/SIDA. Sentencia 02480-2008-PA/TC, caso Ramón Medina Villafuerte, que ordena al Seguro Social de Salud (EsSalud) otorgar al accionante atención médica y hospitalización permanente e indefinida, y proceda a la provisión constante de los medicamentos necesarios para el tratamiento de su enfermedad mental, así como a la realización de exámenes periódicos, y la Sentencia 7231-2005-PA/TC, caso Javier García Cárdenas, que dispone a EsSalud continuar prestando servicio de diálisis al demandante, hasta que no se resuelva lo contrario por disposición de funcionario competente, mediante resolución debidamente motivada.

No obstante, todo cambio de paradigma siempre implica dificultades que pueden solucionarse desde diferentes perspectivas. Por un lado, el reconocimiento del ámbito de derecho fundamental de las personas del derecho a la salud, ha generado su judicialización. Santiago Pereira Campos ${ }^{(18)}$, explica que: (...) la situación se ha vuelto de 
extrema gravedad en Brasil, Colombia y Costa Rica. Es también preocupante en Argentina. En Chile y Uruguay el fenómeno es más incipiente, pero igualmente relevante... En Argentina se discuten dos casos, uno referente a un niño con enfermedad crónica que el sistema decidió no seguir brindando medicamentos, y que luego del fallo judicial se resolvió que el estado debe de garantizar la provisión del mismo al menor; por otro lado, el caso de una madre indigente con ocho hijos, donde una hija padecía de una cardiopatía la cual debía ser operada, y que el jurado decidió que debe de operarse; y casos más recurrentes de medicina prepagada, supeditada al vínculo laboral del trabajador -que pierde prestaciones de salud al terminar el vínculo laboral-, donde un actor con diagnóstico de VIH consiguió, mediante disposición de la corte judicial, que la empresa de medicina prepagada debería mantener la cobertura del plan de salud ${ }^{(19)}$

\section{EXPERIENCIA DE SUSALUD}

En el ámbito de la salud, la regulación gubernamental es una institución formal y cumple la función pública de restringir actividades de los agentes que participan en el mercado, de manera intencional, garantizando así el bien común. Se manifiesta en normas, leyes, reglamentos, decretos o resoluciones, que son de dominio público y obligatorio para todos los agentes, cuyo incumplimiento puede acarrear sanciones. Además, las normas buscan corregir problemas específicos que impiden el desarrollo de los mercados o producen resultados socialmente indeseables ${ }^{(5)}$. En el Perú, en la esfera de derecho programático, se ha consolidado en SUSALUD el registro de todas las IPRESS, IAFAS y UGIPRESS, sobre las cuales se ejerce fiscalización a nivel nacional, a través de supervisiones de campo y seguimiento, monitoreo de la información y vigilancia orientada a verificar el cumplimiento de la normativa relativa a las plataformas de atención ${ }^{(9)}$.

Su principal objetivo es mitigar la probabilidad de que ocurra un evento con consecuencias negativas en el normal desempeño de las actividades administradas, bajo una orientación de gestión del riesgo, como proceso que consiste en identificar, medir, monitorear, comunicar, tratar y controlar los peligros a los cuales se está expuesto, a fin de reducir la probabilidad de su ocurrencia y amortiguar su impacto para el logro de los objetivos de la institución supervisada. Además, se aplican medidas de seguridad de ejecución inmediata, para salvaguardar la salud y la vida de la población, tales como el cierre temporal o definitivo de IPRESS y las que resulten necesarias, a fin de evitar que las IAFAS, IPRESS o UGIPRESS, vulneren el derecho a la salud de los ciudadanos. Igualmente, el regulador emite normas que aseguran el correcto funcionamiento del sistema y la protección del derecho a la salud de los consumidores y usuarios de los servicios de salud ${ }^{(9,10)}$.

En la esfera del derecho a la salud como derecho fundamental de las personas, en SUSALUD se reciben las disconformidades que plantean los consumidores y usuarios de los servicios de salud, sobre aquellas acciones de las IPRESS o IAFAS que pudieran afectarlos. SUSALUD ejerce su función por medio de diferentes mecanismos: la recepción de quejas, las intervenciones de oficio, y el Programa de promoción y protección de los derechos en salud, y la conformación de juntas de usuarios de los servicios de salud.

Por un lado, las quejas comprenden denuncias realizadas directamente a la institución (primer grado); y denuncias que corresponden a reclamos presentados previamente a la IPRESS o IAFAS, que no hayan sido respondidas en el plazo regulado (segundo grado); este último también incluye desviaciones del procedimiento o respuestas no satisfactorias. Otro mecanismo para la atención de quejas son las intervenciones de oficio, que se caracterizan por acciones directas del regulador, sin mediar denuncia, a fin de instaurar investigación ante presuntas vulneraciones, conocidas por medios de comunicación o terceros. Así mismo, SUSALUD cuenta con el Programa de Promoción y Protección de los Derechos en Salud, a través de la presencia de delegados instalados en las IPRESS, que atienden quejas, reclamos y consultas de los usuarios, en el mismo lugar donde se brinda el servicio de salud, interponiendo sus buenos oficios, logrando la solución inmediata de problemas o elevando el asunto al área de "respuesta rápida", para la correspondiente evaluación. Finalmente, para casos referentes a denuncias por negligencia médica, se procede a la respectiva investigación regular.

Otro canal que interviene en la identificación y solución de quejas, está constituido por juntas de usuarios de servicios de salud lideradas por SUSALUD. Éste representa un mecanismo mediador y de diálogo entre usuarios de servicios y SUSALUD, con la finalidad de canalizar propuestas de mejora de los servicios.

Frente a otros sistemas que promueven la judicialización de la salud, SUSALUD tiene potestad sancionadora sobre toda acción u omisión que afecte el derecho a la salud de las personas y la cobertura de su aseguramiento, o los estándares de acceso, calidad, oportunidad, disponibilidad y aceptabilidad de los servicios de salud. Esta se aplica a las IPRESS, IAFAS y UGIPRESS, concentrado así una función antes dispersa en las direcciones regionales de salud, las direcciones de salud, SUNASA e INDECOPI (10).

La figura 1, muestra la distribución de las recomendaciones de inicio de proceso administrativo sancionador (PAS) en SUSALUD durante el periodo enero 2015 a abril de 2016. De éstas solamente requirieron evaluación de inicio 123 , es decir, se ha identificado la falta y se encuentra en la elaboración de los elementos de prueba para su proceso. Otras 87 se encuentran en subsanación debido a que requiere mejorar elementos de prueba para el inicio del PAS y son enviados a las unidades que prepararon los elementos de prueba para complementación. En total 191 fueron PAS iniciadas. De este grupo, 69 han sido resueltos, 38 con responsabilidad y 31 sin responsabilidad. Este 


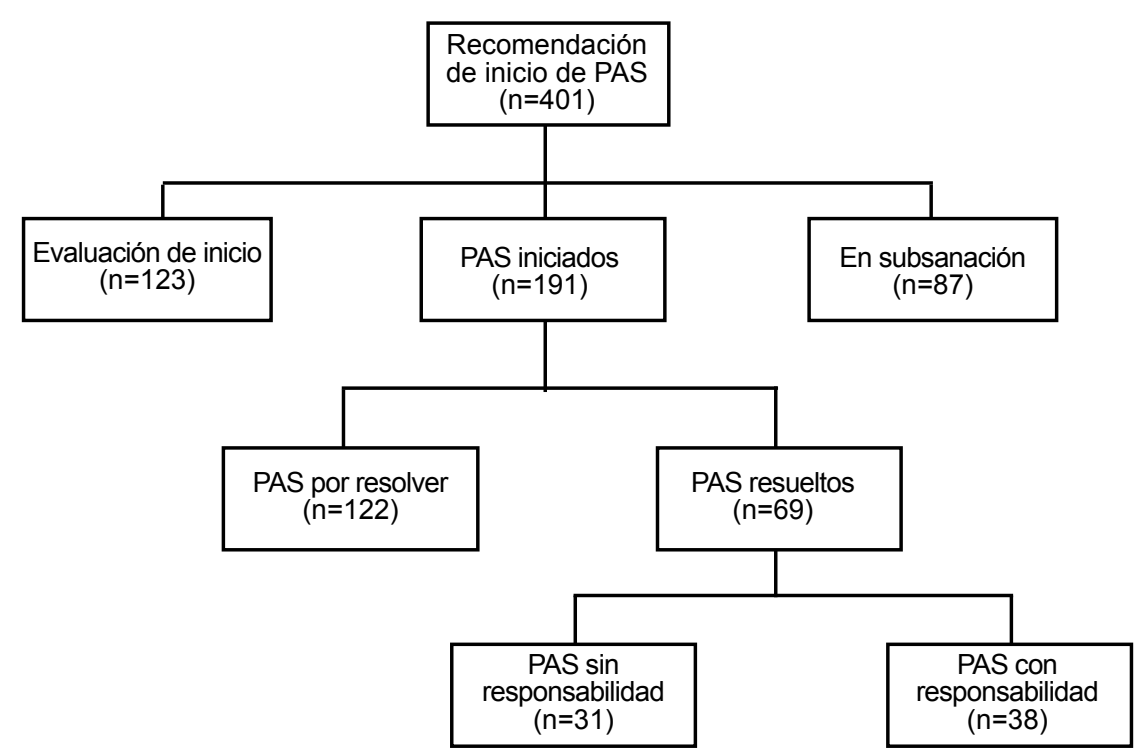

PAS:proceso administrativo sancionador

Fuente: Superintendencia Nacional de Salud

Figura 1. Distribución de las recomendaciones de inicio de PAS en la Superintendencia Nacional de Salud entre enero de 2015 a abril de 2016

proceso guarda correspondencia y es semejante a los otros procesos contenciosos administrativos en el Perú ${ }^{(20)}$.

En un análisis exhaustivo acerca de la distribución de las actividades de fiscalización y sanción del PAS presentado en la Tabla 1, se observa que del total de PAS evaluados, el $28,7 \%$ se encuentran en fase de inicio de PAS; $22,4 \%$ se encuentran observados; $22,2 \%$ se encuentran en fase de instrucción, y $17,2 \%$ son PAS resueltos.

Con respecto al tipo de PAS, el 64,3\% fueron PAS puros, es decir, detectados y supervisados por SUSALUD. En este tipo de PAS el proceso y sanción será efectuado entre SUSALUD y el administrado IAFAS o IPRESS. Por otro lado, un $35,7 \%$ fueron PAS trilaterales ${ }^{(9)}$, es decir, donde interviene SUSALUD, el administrado, y además participa al ciudadano denunciante.

Respecto al tipo de administrado, el $78,3 \%$ de las recomendaciones de inicio de PAS fueron presentadas para IPRESS, 21,2\% por IAFAS y solo 0,5\% por UGIPRESS. Finalmente, en relación al tipo de procedimientos el 72,1\% de éstos son para PAS, es decir, encargados para este proceso y el $27,9 \%$ corresponde al proceso administrativo trilateral (PAT), es decir, procedimientos realizados por SUSALUD, administrado y ciudadano.

Salvo Colombia, que tiene un sistema judicializado ${ }^{(6)}$, no hemos encontrado evaluaciones de entidades fiscalizadoras y sancionadoras de temas de salud en países latinoamericanos. Como una herramienta de proceso el sistema de fiscalización y sanción se encuentra en desarrollo, durante su instalación ha tenido que iniciar un proceso de comunicación y difusión a sus administrados para prevención de sanciones, ello ha sido derivado a cada área que recolecta la

Tabla 1. Distribución porcentual de las actividades de fiscalización y sanción. Superintendencia Nacional de Salud 2015-2016

\begin{tabular}{|c|c|c|}
\hline & $\mathbf{n}$ & $\%$ \\
\hline \multicolumn{3}{|l|}{ Estado } \\
\hline Fase inicio de PAS & 115 & 28,7 \\
\hline Observado & 90 & 22,4 \\
\hline Fase de instrucción & 89 & 22,2 \\
\hline Resuelto & 69 & 17,2 \\
\hline Fase cierre de instrucción & 20 & 5,0 \\
\hline Fase resolutiva & 18 & 4,5 \\
\hline \multicolumn{3}{|l|}{ Tipo de PAS } \\
\hline Puro & 258 & 64,3 \\
\hline Trilateral & 143 & 35,7 \\
\hline \multicolumn{3}{|l|}{ Tipo de administrado } \\
\hline IPRESS & 314 & 78,3 \\
\hline IAFAS & 85 & 21,2 \\
\hline UGIPRESS & 2 & 0,5 \\
\hline \multicolumn{3}{|l|}{ Procedimiento } \\
\hline PAS & 289 & 72,1 \\
\hline PAT & 112 & 27,9 \\
\hline Total & 401 & 100,0 \\
\hline
\end{tabular}

PAS: proceso administrativo sancionador, IPRESS: institución prestadora de servicios de salud, IAFAS: institución aseguradora de fondos de aseguramiento en salud, UGIPRESS: unidad de gestión de instituciones prestadoras de servicios de salud, PAT: proceso administrativo trilateral ${ }^{1}$ Hasta abril de 2016

Fuente: Superintendencia Nacional de Salud 
información dentro de la intendencia, debido a que la mayoría de las recomendaciones de inicio de PAS $(64,3 \%)$ son realizadas por las unidades operativas de SUSALUD desde el 2015, como resultado inicialmente se presentan inicios de PAS no por desconocimiento de los administrados, sino por faltas de otro tipo.

\section{CONCLUSIONES}

El proceso fiscalizador y sancionador en el Perú no se presenta judicializado, a raíz de ser considerado un derecho programático y no fundamental. Esta diferencia respecto a otros países de la región ha llevado a SUSALUD a aplicar otro paradigma en la fiscalización y la sanción, basado en la gestión de riesgos, incluyendo los procesos de quejas, intervenciones de oficio, promoción de derechos, junta de usuarios y en caso de infracciones a la norma (leves, moderadas y graves) el PAS, donde identifica la responsabilidad e impone la sanción.

Fuentes de Financiamiento: autofinanciado

Conflicto de intereses: los autores declaran no tener conflicto de intereses

Contribuciones de autoría: $\mathrm{OQC}$ participó en la concepción y diseño del artículo; redacción del artículo; aprobación de la versión final; asesoría estadística; asesoría técnica o administrativa. OMG participó en el diseño del artículo, redacción del artículo, revisión crítica del artículo, y asesoría estadística. Ambos autores aprobaron la versión final del manuscrito.

\section{REFERENCIAS BIBLIOGRÁFICAS}

1. Oliveira RR, Mangeon PE. Conceitos de regulação em saúde no Brasil. Rev Saude Publica. 2012;46(3):571-6.

2. Robles L. Ejercicio de la función de regulación de la autoridad de salud nacional: eje de la rectoria sectorial en salud. An Fac Med. 2013;74(1):43-8.

3. Muñoz F, López-acuña $D$, Halverson $P$, Guerra de Macedo C, Hanna W, Larrieu $\mathrm{M}$, et al. Las funciones esenciales de la salud pública: un tema emergente en las reformas del sector de la salud. Rev Panam Salud Publica. 2000;8(5):12634.

4. Carpizo J. Los derechos humanos: naturaleza, denominación y caracteristicas. Cuest Const [Internet]. 2011 diciembre [citado el 18 de julio de 2016];25: [27 pág.]. Disponible en: http://www.scielo.org.mx/pdf/cconst/ n25/n25a1.pdf

5. Restrepo J, Rodriguez S. Diseño y experiencia de la regulación en salud en Colombia. Rev Econ Inst. 2005;7(12):165-90.

6. Copetta C, Barrera M. Financiamiento, Regulación y Fiscalización en Latinoamérica: más calidad y derechos en Salud. IV Congreso Latinoamericano de Organos Reguladores y de Control de los Sistemas de Salud; Diciembre de 2011; Santiago, Chile. Santiago: Ministerio de Salud de Chile: 2011. p. 140.

7. ans.gov.br [Internet]. Rio de Janeiro: Agencia Nacional de la Salud Suplementaria; 2016 [citado el 18 de julio de 2016]. Disponible en: http:// www.ans.gov.br/el-sector/regulacion/ fiscalizacion

8. Benavides P, Castro R, Jones I. Sistema público de salud, situación actual y proyecciones fiscales 2013-2050. Santiago: DIPRES; 2013.

9. Perú, Ministerio de Salud. Aprueban Reglamento de Infracciones y Sanciones de la Superintendencia Nacional de Salud - SUSALUD. Lima: MINSA; 2014.

10. Decreto Supremo 008-2014SA. Aprueban el Reglamento de Organización y Funciones de la Superintendencia Nacional de Salud. (El Peruano, número 524956, de 10-062014).

11. Organización de las Naciones Unidas. Declaración Universal de Derechos Humanos. París: ONU; 1948.

12. Comisión Nacional de los Derechos Humanos M. Pacto Internacional de Derechos Económicos, Sociales y Culturales, y su protocolo facultativo. México, D.F.: CNDH; 2012.

13. Alcántara G. La definición de salud de la Organización Mundial de la Salud y la interdisciplinariedad. Sapiens. 2008;9(1):93-107.

14. Ferrajoli L. Sobre los derechos fundamentales. Cuest Const. 2006;(15):113-36.

15. Tribunal Constitucional del Perú. Código Procesal Constitucional (Ley № 28237). Lima: TC; 2004.

16. Tribunal Constitucional de España. Sentencia 35/1996 de 11 de marzo de
1996 (BOE num 93 del 17 de abril de 1996) [Internet]. Madrid: Tribunal Constitucional de España; 1996 [citado el 18 de julio de 2016]. Disponible en: http:// hj.tribunalconstitucional.es/es/Resolucion/ Show/3087

17. República del Perú. Sentencia del Tribunal Constitucional José Luis Correa Condori. Lima. EXP 20162004-AA/TC. 2016. Disponible en: http://www.tc.gob.pe/ jurisprudencia/2005/02016-2004-AA. html.

18. Pereira S. Los Dilemas que plantea la judicialización del derecho a la salud en relación a medicamentos y otras prestaciones de alto costo [Internet]. Santiago: ICHDP; 2013 [citado el 18 de julio de 2016]. Disponible en: http:// www.ichdp.cl/los-dilemas-que-planteala-judicializacion-del-derecho-a-lasalud-en-relacion-a-medicamentos-yotras-prestaciones-de-alto-costo/

19. Abramovich V, Pautassi L. El derecho a la salud en los tribunales. Algunos efectos del activismo sobre el sistema de salud en Argentina. Salud Colect. 2008;4(3):261-382.

20. Ley que regula el proceso contencioso administrativo. Ley 27584. (22 de noviembre de 2001).

Correspondencia: Oscar Quijano-Caballero Av. Velasco Astete 1398 Lima, Perú Teléfono: (511) 3726127 anexo 5818 Correoelectrónico:oquijano@susalud.gob.pe 